\title{
InSb Photodiodes for Monolithic Active Focal Plane Arrays on GaAs Substrates
}

\author{
Vincenzo Pusino, Chengzhi Xie, Ata Khalid, Member IEEE, Matthew J. Steer, Marc Sorel, \\ Iain G. Thayne, and David R. S. Cumming, Fellow, IEEE
}

\begin{abstract}
We describe the development and optical characterization of a planar medium infrared (mid-IR) InSb photodetector on a GaAs substrate technology, capable of integrating MESFETs, to demonstrate a new active pixel device architecture. Our results pave the way for the development of integrated mid-IR focal plane array circuits on a single chip. Device structures with areas down to $0.0016 \mathrm{~mm}^{2}$ were investigated. By deploying a silicon nitride passivation layer, we were able to reduce leakage current in reverse bias by up to $27 \%$ to yield an improved rectifier. Extensive optical characterization was carried out in the near- and mid-IR wavelength range. Responsivities of up to $3.54 \mathrm{~A} / \mathrm{W}$ and quantum efficiency values above unity were obtained in the near-IR range as a consequence of illumination above the bandgap causing impact ionization. In the mid-IR range, responsivities of up to $0.97 \mathrm{~A} / \mathrm{W}$ were observed. The bandwidth of the devices proved compatible with video-rate standard sampling rates.
\end{abstract}

Index Terms-Focal plane arrays (FPAs), GaAs, infrared image sensors, InSb, mid-infrared (mid-IR), monolithic integration.

\section{INTRODUCTION}

$\mathbf{S}$ ENSING and imaging in the medium-infrared (mid-IR) wavelength range is increasingly important for defense and security applications, environmental monitoring, astronomy, gas sensing, and medical diagnostics [1]. Mid-IR detection has been demonstrated with several antimonide-based III-V semiconductor materials, such as InSb, InAlSb, InAsSb, and, more recently, InAs/GaSb superlattice structures [2]-[5]. InSb in particular has considerable potential: its sensitivity spans optical wavelengths from 1.5 to $7 \mu \mathrm{m}$ at room temperature, a spectral range where absorption lines of many gases (NO, $\mathrm{CO}, \mathrm{CO}_{2}$ ) lie. This makes InSb an ideal candidate for gas sensing applications, and InSb gas detectors have been demonstrated and commercialized [6]. In order to make an imaging device such as a focal plane array (FPA), a system capable of individual addressing and read-out of the

Manuscript received March 4, 2016; revised May 9, 2016 and June 1, 2016; accepted June 6, 2016. Date of publication June 21, 2016; date of current version July 21, 2016. This work was supported by the Engineering and Physical Sciences Research Council under Grant EP/J018678/1 and Grant EP/M01326X/1. The review of this paper was arranged by Editor E. G. Johnson. (Corresponding author: Vincenzo Pusino.)

The authors are with the Electronics and Nanoscale Division, School of Engineering, University of Glasgow, Glasgow G12 8LT, U.K. (e-mail: vincenzo.pusino@glasgow.ac.uk; c.xie.1@ research.gla.ac.uk; ata.khalid@ glasgow.ac.uk; matthew.steer@glasgow.ac.uk; marc.sorel@glasgow.ac.uk; iain.thayne@glasgow.ac.uk; david.cumming.2@glasgow.ac.uk).

Color versions of one or more of the figures in this paper are available online at http://ieeexplore.ieee.org.

Digital Object Identifier 10.1109/TED.2016.2578982
InSb photodiodes (PDs) is necessary. In the visible wavelength range, this can be achieved through monolithic integration of photodetectors with MOSFETs on a CMOS silicon platform. The small bandgap materials used for mid-IR imaging make monolithic integration of photodetectors and addressing circuitry challenging, given the difficulty in implementing transistors on such materials. FPAs manufactured so far are thus based on hybrid designs where the detectors and a CMOS read-out integrated circuit (ROIC) are fabricated separately and subsequently bonded using flip-chip technology [7]-[9]. This hybrid technology requires substrate thinning and additional steps that complicate device fabrication and incur a significantly increased cost. The fact that antimonide-based FPAs require cooled operation near $80 \mathrm{~K}$ can be problematic for hybrid designs: the large mismatch in thermal expansion coefficients between antimonide materials and silicon limits the range of operation and storage temperatures. This is because thermal cycles with a big temperature differential can cause connection failure and cracking of the semiconductor [10]. The greatest advantage of hybrid FPAs, however, is to allow separate optimization of detector and ROIC.

Recent work has demonstrated a monolithically integrated active mid-IR pixel combining an InSb-based mid-IR detector and a GaAs MESFET [11]. However, there are still challenges to overcome toward the development of InSb monolithic FPAs. In this paper we describe the optimization of planar InSb photodetectors on a GaAs substrate and their electrical and optical performances. We demonstrate successful implementation of high-responsivity InSb PDs capable of supporting the videorate bandwidths necessary for mid-IR FPAs. The devices were achieved on a novel architecture suitable for integration of InSb PDs and underlying MESFETs, with anode and cathode contacts on the front-side of the wafer. In order to attain our goal, we developed a robust process so that the InSb devices could sustain subsequent processing while avoiding device failure. This was done by adapting traditional techniques, such as passivation, to protect devices both physically and in terms of electrical performance.

In Section II, we describe the epistructure of the material used and the fabrication flow for implementing the InSb PDs. Section III presents the results obtained from the electrical characterization of the InSb photodetector in terms of $I-V$ characteristics at 77 and $300 \mathrm{~K}$. Section IV reports on the optical characterization carried out. Section V discusses and summarizes the main findings, and conclusions are drawn in Section VI. 


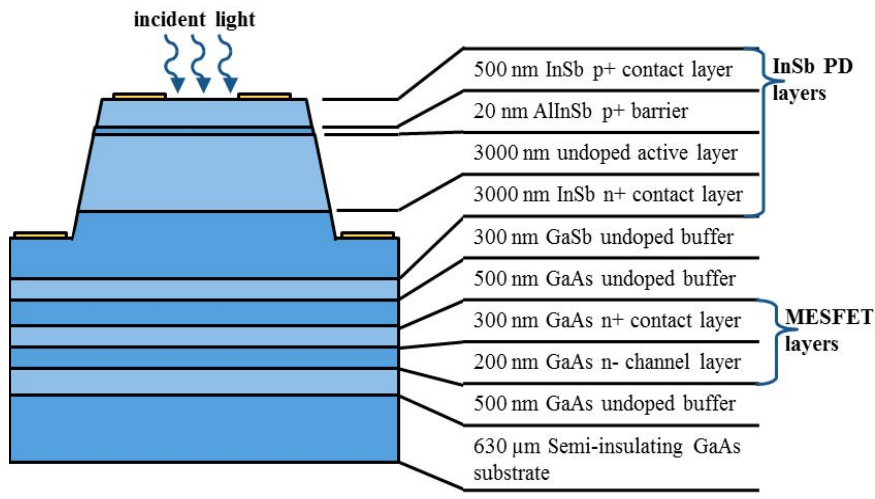

Fig. 1. Material structure, detailing composition, doping type, and thickness of the layers.

\section{DEVICES}

The material used consists of an integrated structure including a p-i-n InSb detector and a GaAs MESFET to be used for photocurrent signal read-out. The epi-structure was grown on a semi-insulating (SI) GaAs substrate by molecular beam epitaxy (MBE) growth. Successful multilayer MBE growth is more challenging on SI substrates than on doped ones due to temperature instability across the wafer [12]. A GaAs substrate was preferred to silicon as it presented lower lattice mismatch to InSb (14.5\% instead of 19\%). Using GaAs also allows for consistent use of III-V process technology for the completed device architecture. In order to ease the strain that would result from direct growth of InSb on GaAs, a 300-nm buffer of gallium antimonide ( $\mathrm{GaSb}$ ) was inserted between the InSb and the GaAs layers. The complete epitaxial structure, depicted in Fig. 1, consists of (bottom to top): 1) an undoped GaAs buffer; 2) a lightly n-doped layer; 3) a heavily n-doped layer; and 4) again a GaAs buffer. These first layers constitute the GaAs MESFET, separated from the above InSb PD by the buffer. The PD layers, again bottom to top, are: 1) a highly n-doped $\left(1 \times 10^{18}\right)$ contact layer; 2$)$ a nonintentionally doped layer, where absorption of the mid-IR radiation takes place; 3 ) an $\mathrm{Al}_{0.15} \mathrm{In}_{0.85} \mathrm{Sb}$ barrier, also highly doped, but p-type; and 4) finally another highly doped $\left(1 \times 10^{18}\right)$ p-type contact layer. Beryllium and tellurium were used as p-type and n-type dopants, respectively, for the antimonide layers. Silicon was the n-type dopant for the GaAs MESFET layers. The $\mathrm{Al}_{0.15} \mathrm{In}_{0.85} \mathrm{Sb}$ barrier is there to ensure a more rectifying device behavior, by preventing drift of the thermally generated carriers to the contact region, where they would recombine and contribute to noise [13]. Fig. 1 shows the etch depth and profile necessary to reach the InSb $\mathrm{n}+$ contact and fabricate a working InSb PD.

Fabrication on InSb poses some constraints, especially with regard to processing temperatures and plasma damage that can cause increased leakage and short circuiting of the devices [14]-[17]. For these reasons, all processes used in this work have been carried out at temperatures below $200{ }^{\circ} \mathrm{C}$, and ashing and descum steps were carried out with low levels of $\mathrm{RF}$ power in order to minimize plasma-induced damage and heating of the sample.

The fabrication flow of the InSb PDs was as follows. The first step was the definition of the mesas using a standard

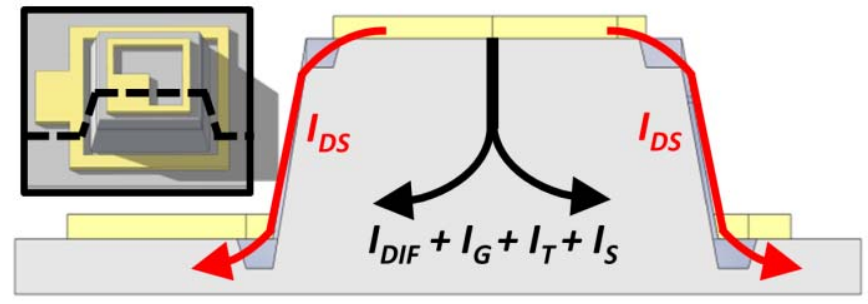

Fig. 2. Cross section of an InSb PD (inset) highlighting the native oxide as well as the current paths of bulk and surface leakage.

photoresist mask (1.8 $\mu \mathrm{m}$ of Shipley Microposit S1818 series Photoresist, patterned through UV exposure and development in Microposit MF-319) to allow the pattern to be transferred onto the material by a subsequent wet etch step. We used a solution of $\mathrm{C}_{6} \mathrm{H}_{8} \mathrm{O}_{7}: \mathrm{H}_{2} \mathrm{O}_{2}: \mathrm{H}_{2} \mathrm{O}$ at the ratio $33: 2: 80$ by weight to etch the InSb mesas, since this provided an easily controllable isotropic etch with a room temperature etch rate varying between 15 and $20 \mathrm{~nm} / \mathrm{min}$ [18]. The targeted etch depth was $3.5 \mu \mathrm{m}$. Mesas with several different areas were made, from $0.64 \mathrm{~mm}^{2}\left(800 \times 800 \mu \mathrm{m}\right.$ square mesa) to $0.0016 \mathrm{~mm}^{2}(45-\mu \mathrm{m}$ diameter circular mesa). Following wet etch and resist strip, ohmic contacts could immediately be made on unpassivated devices. Contacts were made using Ti/Au evaporation followed by lift-off through a bilayer of resist. Thermal annealing of the contacts was not necessary due to the small bandgap of $\mathrm{InSb}(0.172 \mathrm{eV}$ at $300 \mathrm{~K})$.

To investigate the effect of passivation, several devices were coated with silicon nitride $\left(\mathrm{SiN}_{x}\right)$, since it could be deposited at low temperature $\left(<40{ }^{\circ} \mathrm{C}\right)$ using an Oxford Instruments System 100 inductively coupled plasma 180 tool. Since the devices have mesas with a $3.5-\mu$ m etch depth a 400 -nm-thick $\mathrm{SiN}_{x}$ layer was used to ensure uniform sidewall coverage. For devices passivated in this way, windows in the $\mathrm{SiN}_{x}$ layers were etched prior to ohmic contact formation using sulphur hexafluoride $\left(\mathrm{SF}_{6}\right)$ plasma in an Oxford Instrument Plasmapro 100 etching tool. Ohmic contacts were then made using the same process already described.

\section{ElectricAl CharacterizATION}

Under the condition where there is no illumination, the current flow under reverse bias has several components [19]

$$
I_{D}=I_{\mathrm{DIF}}+I_{\mathrm{DS}}+I_{G}+I_{T}+I_{S}
$$

where $I_{D}$ is the dark current, $I_{\mathrm{DIF}}$ is the diffusion current in the material, $I_{\mathrm{DS}}$ is the surface diffusion current (see Fig. 2), $I_{G}$ and $I_{T}$ are parasitic bulk currents arising from, respectively, carrier generation-recombination and tunneling, and finally, $I_{S}$ is the shunt current contribution related to leakage paths caused by defects such as threading dislocations. The current components in (1) are dependent on the applied voltage and temperature of the devices.

$I_{\mathrm{DS}}$ arises from the unwanted conductivity occurring in the native oxide on the sidewall surface. $I_{\mathrm{DIF}}, I_{G}, I_{T}$, and $I_{S}$ all scale linearly with the area of the devices, as does the photogenerated current when illuminated. However, $I_{\mathrm{DS}}$ is associated only with the exposed sidewall surface of 


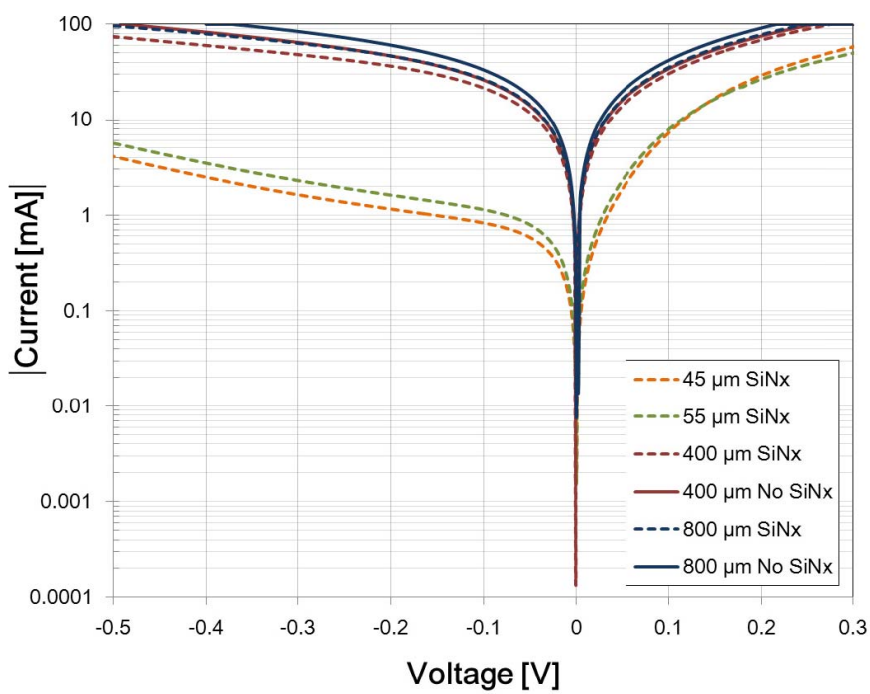

Fig. 3. Room temperature magnitude of current versus voltage characteristics of square InSb PDs of various sizes with (dashed lines) and without (continuous lines) $\mathrm{SiN}_{x}$ passivation applied.

the device, and as we show, it can be significantly reduced by using $\operatorname{SiN}_{x}$ as a surface passivation layer. The smallest device studied in this paper had a $45-\mu$ m-diameter mesa. Since the leakage component $I_{\mathrm{DS}}$ becomes an increasingly large component of $I_{D}$ as device mesas are reduced in area, successful passivation is necessary.

$I-V$ characterization was carried out at room temperature using dc probes connected to an Agilent 4155C semiconductor parameter analyzer. The improvement in $I_{\mathrm{DS}}$ with $\mathrm{SiN}_{x}$ passivation was confirmed from the experimental results, shown in Fig. 3. Devices without $\mathrm{SiN}_{x}$ passivation (solid lines) were found to have the least rectifying behavior and the highest values of dark current. For PDs with $400 \mathrm{~nm}$ of $\mathrm{SiN}_{x}$ passivation applied (dashed lines), the measured $I-V$ characteristic at room temperature showed a net improvement, with the $I-V$ curve becoming more rectifying (because of a reduction in $\left.I_{\mathrm{DS}}\right)$ and a decrease in leakage ranging from $16 \%$ to $27 \%$ with respect to the nonpassivated devices. After demonstrating the beneficial effect of $\operatorname{SiN}_{x}$ passivation for $800 \times 800 \mu \mathrm{m}$ and $400 \times 400 \mu \mathrm{m}$ size square PDs, all subsequently fabricated InSb PDs were passivated, and the focus was on the effect of scaling the mesa size. As Fig. 3 shows, there is also a reduction in dark current when the device area is scaled, since the bulk leakage components are minimized (green dashed and orange lines).

The effect of making circular or square devices was explored, since the potential effect of current crowding in the corners of square devices was of concern to us. The relationship between the leakage current for the InSb PDs under reverse bias and the area for circular and square devices is summarized in Fig. 4. Since InSb PDs are usually operated with a small reverse bias, normally below $300 \mathrm{mV}$ in magnitude, our data were taken with a bias of $-50 \mathrm{mV}$. All measurements were made at $300 \mathrm{~K}$. The plot shows that for small devices the current increases linearly with area for both circular and square PDs. For the smallest device we studied, which was a circular PD with a diameter of $45 \mu \mathrm{m}$,

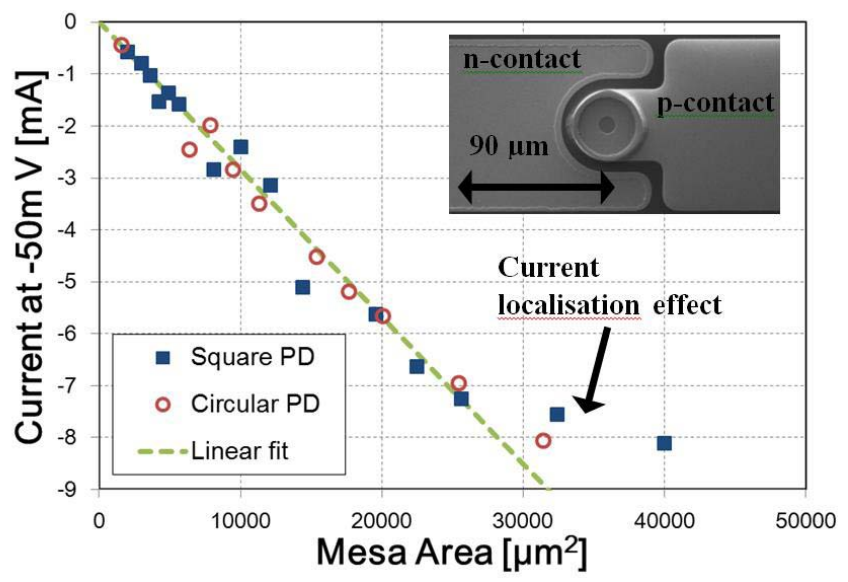

Fig. 4. Evolution of leakage current versus devices area with a bias of $-50 \mathrm{mV}$ applied. Inset: an SEM image of a circular InSb PD.

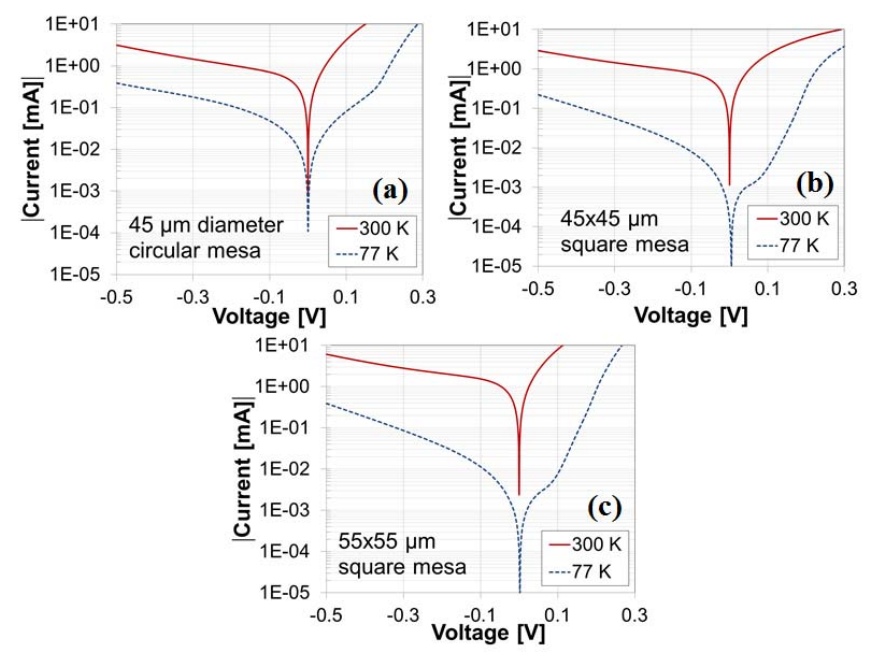

Fig. 5. Comparison between magnitude of current versus voltage curves measured at room temperature (red solid line) and at $77 \mathrm{~K}$ (blue dashed line) for (a) 1590 , (b) 2025 and (c) $3025 \mu \mathrm{m}^{2}$ InSb PDs.

the dark current was approximately $520 \mu \mathrm{A}$ at $-50 \mathrm{mV}$. The current versus area line intersects the current axis at $I \sim 0$, demonstrating that the devices have a very small $I_{\mathrm{DS}}$ component in $I_{D}$. This supports our data that show the passivated devices having low $I_{\mathrm{DS}}$ (see Fig. 3). As the mesa is increased to large sizes, beyond approximately $30000 \mu \mathrm{m}^{2}$, the current begins to saturate. In our devices we use a single annular anode on the top surface of the device (see the inset in Fig. 4). As the device area increases, thermally generated carriers toward the center of the device are not collected by the anode; hence, current scaling saturates for large mesa areas [20]. This effect is more evident in the square mesa devices. The shape of the InSb PD did not appear to influence the dark current. Similar values of dark current were measured from circular and square PDs with the same device area.

For mid-IR sensors it is often desirable to cool the device in order to reduce the unwanted thermally excited current. We have therefore studied the diode $I-V$ characteristics of several devices at 77 and $300 \mathrm{~K}$ for a side-by-side comparison. These data are shown in Fig. 5. 
By comparing the curves at room temperature with those at $77 \mathrm{~K}$, the improvement in rectifying behavior is clear. The leakage current near $0 \mathrm{~V}$ for the $45-\mu \mathrm{m}$ circular mesa [Fig. 5(a)] decreased from $1.14 \mu \mathrm{A}$ at $300 \mathrm{~K}$ to $150 \mathrm{nA}$ at $77 \mathrm{~K}$, and a similar reduction was observed for the bigger mesas [Fig. 5(b) and (c)]. At a reverse voltage bias of $-50 \mathrm{mV}$, the dark current decreases from $\sim 520 \mu \mathrm{A}$ at room temperature to $\sim 3 \mu \mathrm{A}$ for a $45-\mu \mathrm{m}$ diameter circular InSb PD [Fig. 5(a)]. As previously stated, thermally generated carriers provide an important contribution to the current at $300 \mathrm{~K}$ because of the small energy bandgap of InSb, whereas the contribution at $77 \mathrm{~K}$ is much less. Further experiments were carried out at $77 \mathrm{~K}$, by comparing the $I-V$ characteristics of unpassivated and passivated devices. The effect of $\mathrm{SiN}_{x}$ passivation at $77 \mathrm{~K}$ was found to be negligible, as nearly overlapping curves were obtained from unpassivated and passivated InSb PDs at $77 \mathrm{~K}$. This suggests very small $I_{\mathrm{DS}}$ for cryogenically cooled devices. However, as previously mentioned, at higher temperatures $\mathrm{SiN}_{x}$ passivation is necessary for reduced $I_{D}$.

\section{OPTICAL CHARACTERIZATION}

The response of the InSb PDs to infrared light was characterized. For all experiments described in this section, only the $p$ and $n$ contacts of the InSb PDs were connected, and the MESFET contacts, if present, were left floating. The GaAs substrate is SI and the MESFET layers do not interfere with the InSb PD characterization or introduce additional current leakage paths, owing to the thick undoped buffer layer. Measurements were carried out using an Anritsu MG9638A tunable laser with a continuous wave output, the output beam of which was focused on the devices. Unless otherwise stated, all InSb PDs were measured at zero bias, and the current flowing through them was measured directly with a picoammeter (Keithley 487). The total laser power $P_{\text {tot }}$ illuminating the diodes was measured with a broad area power meter (ThorLabs S132C). The photocurrent $I_{\mathrm{PH}}$ was measured for $P_{\text {tot }}$ values ranging from 1 to $10 \mathrm{~mW}$ while the wavelength was set at $1.61 \mu \mathrm{m}$, corresponding to photon energies of approximately 4.5 times the energy bandgap of InSb. For each laser power, the responsivity was calculated with the formula $R_{P}=\left(I_{\mathrm{PH}} / P_{\mathrm{tot}}\right) \cdot\left(A_{\text {beam }} / A_{\mathrm{PD}}\right)$, where $A_{\text {beam }}$ is the area of the laser spot illuminating the diode and $A_{\mathrm{PD}}$ is the mesa area of the measured InSb PD. This formula does not take into account the area of the top contact. $R_{P}$ was calculated for each laser power and then averaged over all power values measured to obtain the average responsivity $\overline{R_{P}}$. Fig. 6 displays the average responsivity $\overline{R_{P}}$ as a function of the measured InSb PDs area, together with the corresponding quantum efficiency.

It can be seen that for small devices the responsivity presents a large spread in the measured values. We attribute this to factors such as fabrication tolerances, bonding wires shadowing, and top contact area differences. For larger mesas a large drop in responsivity is observed. This characteristic is related to the current localization effect already described, which applies to photogenerated carriers as well as thermally excited carriers. For large-area InSb PDs, only the electronhole pairs generated close to the annular top contact contribute to the photocurrent [21]. Most of the photogenerated carriers

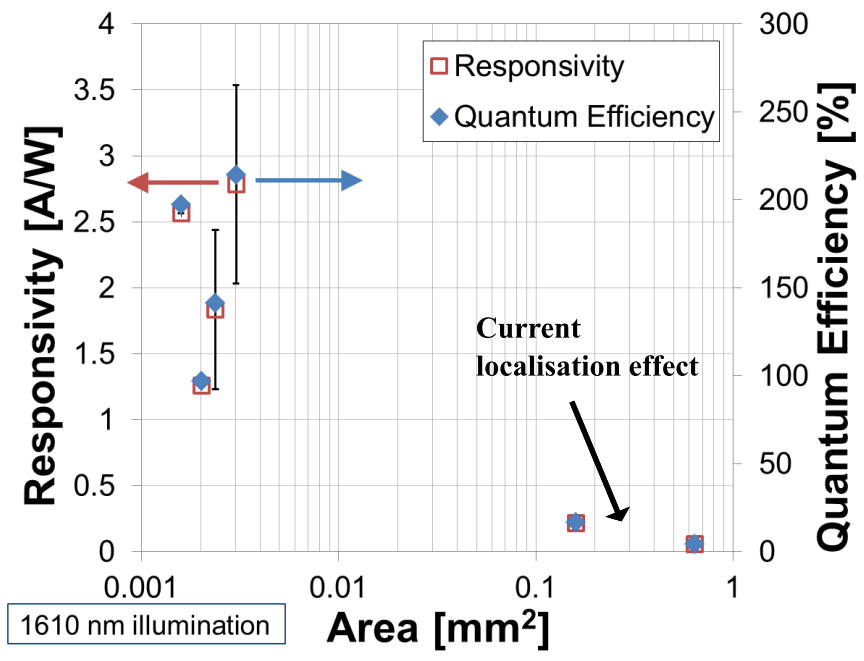

Fig. 6. Responsivity and quantum efficiency of InSb PDs passivated with $400 \mathrm{~nm}$ of $\mathrm{SiN}_{x}$ plotted versus the area of the devices; data obtained for illumination at a wavelength of $1.61 \mu \mathrm{m}$. The devices were measured at zero bias.

recombine; thus, for large areas the majority of the surface does not contribute to the photocurrent. Fig. 6 also depicts the quantum efficiencies calculated for the corresponding responsivities. It can be seen that the obtained values are greater than $100 \%$ for most of the small area detectors. This result can be explained in terms of impact ionization.

The InSb PDs were illuminated with photon energies much higher than the InSb bandgap. Each photon thus generates carriers with enough kinetic energy to produce additional electron-hole pairs [22]. Further experiments confirmed that the application of an electric field indeed enhanced the impact ionization phenomenon. The application of a $50-\mathrm{mV}$ reverse bias on a $45 \times 45 \mu \mathrm{m}$ square InSb PD increased the responsivity from 1.28 to $2.16 \mathrm{~A} / \mathrm{W}$ (and thus the quantum efficiency from $98 \%$ to $166 \%$ ). The increase in responsivity, however, also decreased the signal-to-noise ratio approximately by a factor of 100 because of the increased contribution from thermally generated carriers.

Characterization in the mid-IR spectral range was also carried out with a Fourier transform infrared (FTIR) spectrometer (Bruker Vertex 70). The instrument's built-in midIR detector was replaced with one of the fabricated $\mathrm{InSb}$ PDs, the output of which was used to measure the emission spectrum of the spectrometer mid-IR source (a glow-bar). All characterized devices were measured at room temperature and atmospheric pressure. Fig. 7 shows a typical photocurrent spectrum from one of the devices. The signal drop at $4.2 \mu \mathrm{m}$ is due to absorption from carbon dioxide molecules in the atmosphere.

The data in Fig. 7 demonstrate successful photocurrent generation in the InSb PDs in the mid-IR spectral range.

Responsivity data in the mid-IR wavelength range were also obtained. The optical power of the FTIR source illuminating the InSb PDs was measured with a mid-IR thermal power sensor (ThorLabs S401C), while monitoring their current with the picoammeter. The results, displayed in Fig. 8, show a trend similar to the measurements at shorter wavelengths, with the highest responsivity achieved by the smallest devices and 


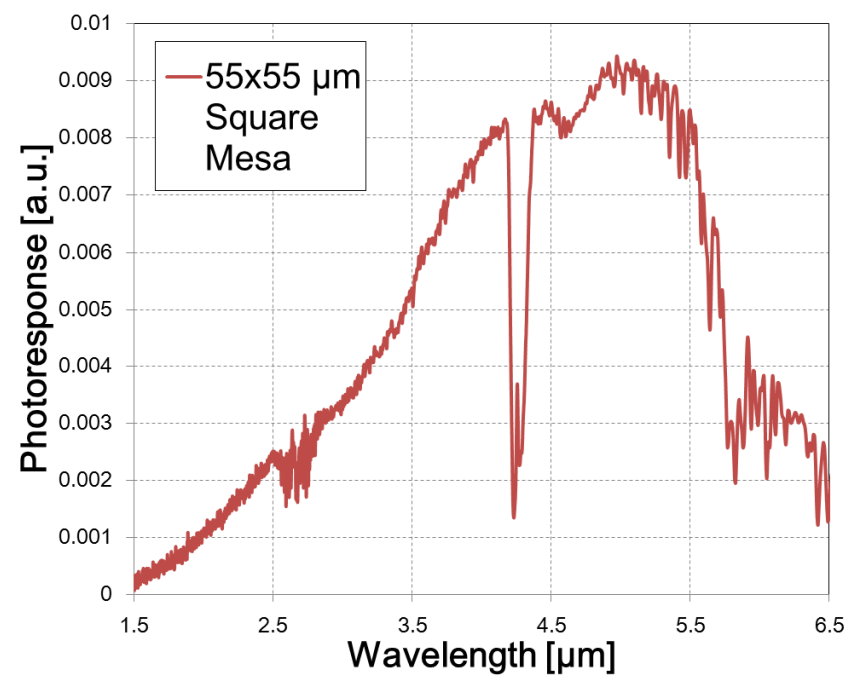

Fig. 7. Typical photocurrent spectrum obtained with an FTIR spectrometer from a $55 \times 55 \mu \mathrm{m}$ square InSb PD at $300 \mathrm{~K}$. The devices were measured at zero bias.

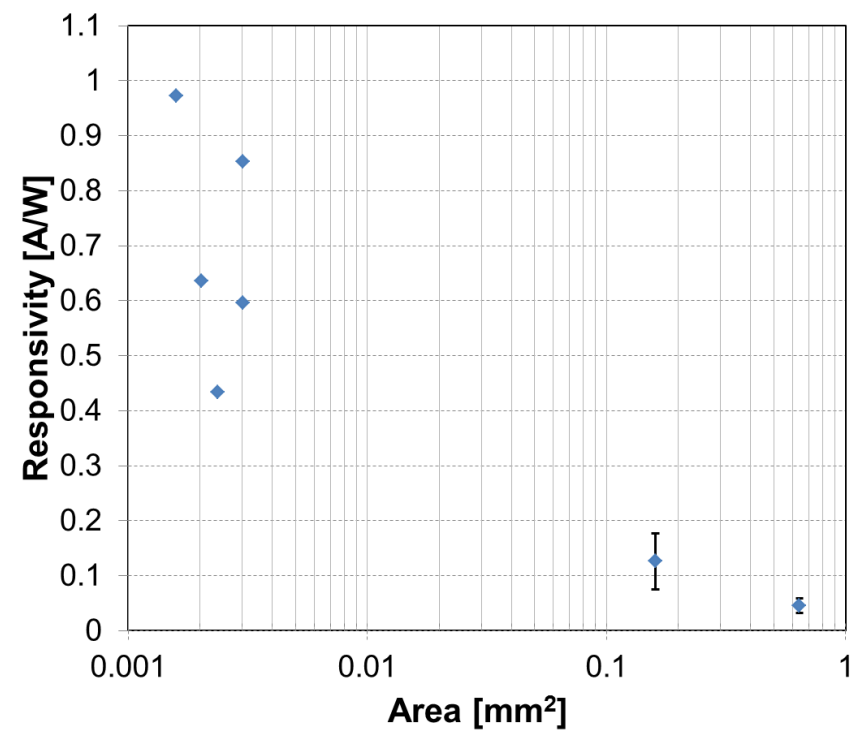

Fig. 8. Responsivity comparison for InSb PDs of varying areas. Illumination was from a glow-bar source, the emission wavelength of which peaked at $2.4 \mu \mathrm{m}$. The devices were measured at zero bias.

the values dropping for increasing area. The maximum value of responsivity measured in the mid-IR range $(0.97 \mathrm{~A} / \mathrm{W}$, corresponding to a quantum efficiency of $50 \%$ ) was obtained from a $45-\mu \mathrm{m}$-diameter circular InSb PD. Since illumination occurs near band-edge, there is no impact ionization taking place and lower values of quantum efficiency are observed. Passivated devices yielded slightly higher responsivity values, once again confirming the advantages of $\mathrm{SiN}_{x}$ passivation.

We characterized both the static and dynamic responses of the PDs to light. Bandwidth measurements were carried out by modulating the continuous wave output of the laser with a Mach Zehnder modulator (model T.MZH1.5-40X-ADC-Y-Z from Sumitomo Osaka Cement Company). The modulator was driven by the sine wave from an arbitrary waveform generator (Agilent 33220A). The modulated light, the frequency of which was varied during the experiments, was shone on

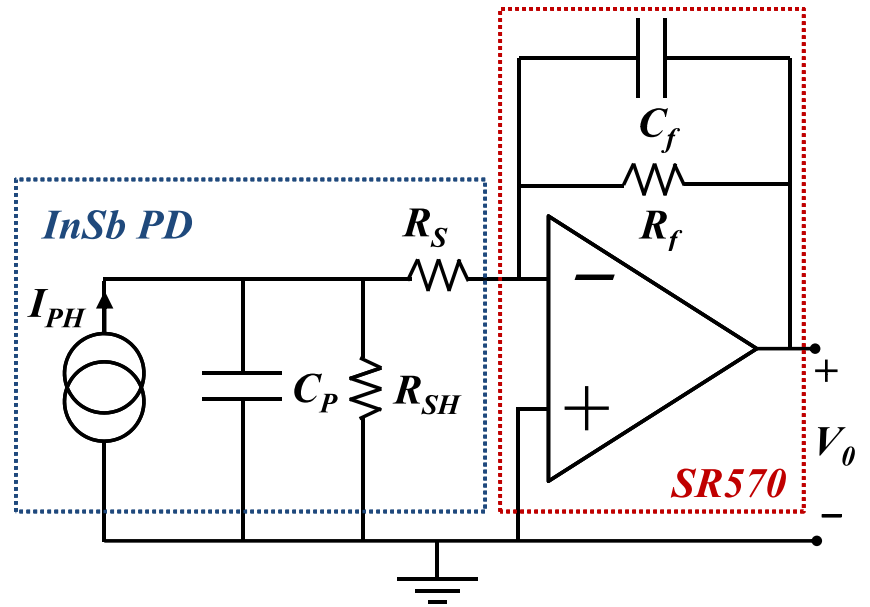

Fig. 9. Equivalent circuit of the InSb PD connected to the current preamplifier.

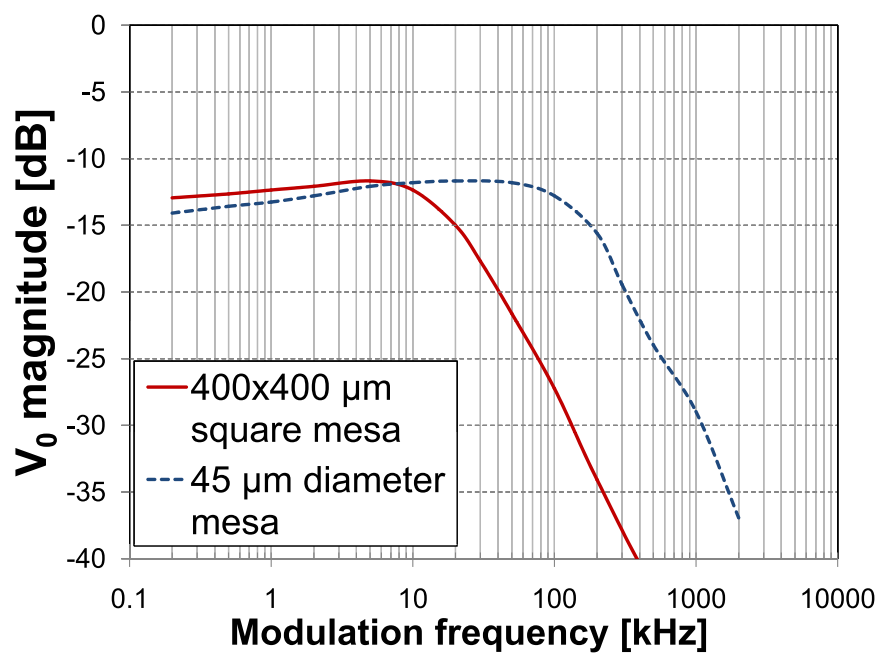

Fig. 10. Bode magnitude plots measured at $300 \mathrm{~K}$ for a large-area (red solid line) and a small-area (blue dashed line) InSb PD, passivated with $400 \mathrm{~nm}$ of $\operatorname{SiN}_{x}$. The devices were measured at zero bias.

the InSb PD under test, which was connected to a low noise transimpedance amplifier (SR570 from Stanford Research Systems) for current-to-voltage conversion. The settings of the SR570 amplifier were chosen so that its bandwidth was $1 \mathrm{MHz}$, in order not to band-limit the measurement. The InSb PDs were measured at zero bias, and after current-tovoltage conversion the amplifier output was recorded with a Rohde \& Schwarz RTO1022 digital oscilloscope. The laser wavelength was set to $1.58 \mu \mathrm{m}$ and the average power of the modulated output beam was set to $10 \mathrm{~mW}$ throughout the experiments. Fig. 9 shows the equivalent circuit of the InSb PD and of the transimpedance amplifier, to support the analysis of the experimental results, which are depicted in Fig. 10.

The transimpedance amplifier in Fig. 9 has a feedback resistor and capacitor $R_{f}$ and $C_{f}$, respectively. The output voltage at $\mathrm{dc}$ is given by $V_{0}=-R_{f} \times I_{\mathrm{PH}}$. Fig. 9 also includes the equivalent circuit of the InSb PD, which consists of a current source for the photogenerated current $I_{\mathrm{PH}}$, the InSb PD capacitance $C_{P}$, and also its shunt and series resistances, $R_{\mathrm{SH}}$ and $R_{S}$, respectively. The series resistance $R_{S}$ of the PD derives from the metal contacts and bonding wires. 
A circular transmission line model pattern [23] was used to determine the resistivity of the contacts, which was found to be $\sim 5.9 \times 10^{-5} \Omega \cdot \mathrm{cm}^{2}$ for the $p$-contacts and $7 \times 10^{-7} \Omega \cdot \mathrm{cm}^{2}$ for the $n$-contacts. For our smallest devices $R_{S}$ is calculated to be $\sim 7 \Omega$, whereas for the larger ones it drops to $\sim 0.3 \Omega$. The transimpedance of the circuit is given by [24]

$$
\frac{V_{0}}{I_{\mathrm{PH}}}=\frac{-R_{\mathrm{SH}}}{R_{S}+R_{\mathrm{SH}}} \cdot \frac{1}{1+j \omega\left(\frac{R_{S} R_{\mathrm{SH}}}{R_{S}+R_{\mathrm{SH}}} C_{P}\right)} \cdot \frac{R_{f}}{1+j \omega R_{f} C_{f}} .
$$

This system has two poles: one related to the PD response at the frequency $f_{0}=2 \pi\left(R_{\mathrm{SH}} \| R_{S}\right) C_{P}$ and the other related to the transimpedance amplifier response at the frequency $f_{1}=2 \pi R_{f} C_{f}$. The dominant pole is $f_{0}$, as $f_{1}$ lies at $1 \mathrm{MHz}$. Fig. 10 displays the measured bandwidth of the system for a large-area $\left(160000 \mu \mathrm{m}^{2}\right)$ and a small-area $\left(\sim 1600 \mu \mathrm{m}^{2}\right) \mathrm{InSb}$ $\mathrm{PD}$; it can be seen that the 3 - $\mathrm{dB}$ cutoff $f_{3} \mathrm{~dB}$ are approximately 20 and $200 \mathrm{kHz}$, for the large- and small-area InSb PD, respectively. The bandwidth measurement therefore allows extraction of the PD capacitance $C_{P}$, which was found to be $33.2 \mu \mathrm{F}$ for the $400 \times 400 \mu \mathrm{m}$ square device and $118.2 \mathrm{nF}$ for the $45-\mu \mathrm{m}$-diameter InSb PD. We measured the capacitance of the small InSb PD with an LCR meter and found it to be $136.5 \mathrm{nF}$, which is in good agreement with the calculated value. Unfortunately, the same comparison could not be carried out for the large device, since its leakage current prevented us from making a reliable capacitance reading from the $L C R$ meter, even at zero bias. Using the data from the bandwidth measurement, we estimate that the capacitance associated with the InSb PD decreases by a factor of $\sim 280$ when the device active area is scaled by approximately 600 times. The discrepancy arises because the capacitance of the InSb PD has two components, one associated with the active area and the other associated with the device perimeter where the contact lies. As devices are made smaller, the component related to the contact area starts to dominate [25].

The measured bandwidth is low compared with that which has been previously reported [26], [27]. However, the maximum values of bandwidth stated in [26] and [27] were obtained with a reverse bias of up to $2.5 \mathrm{~V}$ applied to the PD. We were limited to zero-bias measurements because the SR570 amplifier saturated when the devices were biased; hence, we have not explored this possibility with our devices. The response time of the InSb PDs is also negatively affected by the presence of lattice defects, impurities, and surface states that act as charge trapping centers and introduce charge latency [28]. It is also possible that impurity sites in the passivation layer trap holes which can then tunnel back to the InSb layer [29].

\section{Discussion}

Good electrical and optical performances were obtained from InSb PDs on an SI GaAs substrate including GaAs MESFET layers in the material stack.

We have reported a decrease in dark current ranging from $16 \%$ to $27 \%$ depending on bias by applying a $\mathrm{SiN}_{x}$ passivation technique. We observed that scaling of the InSb PD mesa sizes also reduced leakage. This reduction is due to scaling of the components of the dark current related to parasitics in the bulk of the material. The effect of mesa shape (circular or square) was also assessed, but no correlation was found between this and the leakage current. Our diodes show a good rectifying $I-V$ characteristic at $300 \mathrm{~K}$, comparable to the best InSb PDs reported so far [30]. Our devices are good rectifiers at room temperature even though their area is $\sim 20$ times larger than the InSb PDs reported in [30], and their performance is expected to improve further if they are made smaller. The leakage current density from our smallest device $\left(\sim 1600 \mu \mathrm{m}^{2}\right)$ is $0.7 \mathrm{nA} / \mu \mathrm{m}^{2}$.

Extensive optical characterization was also carried out, measuring responsivities both in the near-infrared and in the mid-IR wavelength range. The former showed that for high photon energies impact ionization takes place and quantum efficiency above unity was achieved. A maximum responsivity of $0.97 \mathrm{~A} / \mathrm{W}$, corresponding to a quantum efficiency of 50\%, was obtained in the mid-IR wavelength range. Optical measurements were also used to characterize the dynamic response of our devices. The highest bandwidth we observed was $200 \mathrm{kHz}$ for a $45-\mu \mathrm{m}$-diameter circular mesa. Despite this value being lower than that which has previously been reported, it is compatible with standard video sampling rates, making our FPA feasible.

\section{CONCLUSION}

We have developed a new mid-IR photodetector on a novel planar device architecture suitable for next-generation monolithic mid-IR FPAs. Due to recently emerging interest in mid-IR imaging technologies, the design of FPAs capable of offering good performance at room temperature while providing compactness, as well as portability and cost reduction, is actively sought. We have shown a systematic characterization of InSb PDs fabricated on an integrated platform which will allow addressing through GaAs transistors. These findings represent a major step toward future integrated FPA chips based on monolithic integration of InSb photodetectors and GaAs switching transistors.

Future efforts will focus on the demonstration of working arrays of InSb-based photopixels addressed by GaAs MESFETs. Further investigations will be carried out to improve noise and bandwidth performance of our devices.

\section{ACKNOWLEDGMENT}

The authors would like to thank all the staff at the James Watt Nanofabrication Centre (JWNC) for their assistance. All the fabrication described in this paper was carried out at JWNC, University of Glasgow.

\section{REFERENCES}

[1] A. Krier, Mid-Infrared Semiconductor Optoelectronics. London, U.K.: Springer, 2006

[2] N. Kuze et al., "High performance miniaturized InSb photovoltaic infrared sensors operating at room temperature," J. Crystal Growth, vols. 301-302, pp. 997-1000, Apr. 2007, doi: $10.1016 /$ j.jcrysgro.2006.11.179.

[3] A. Rogalski, "InAs ${ }_{1-x} \mathrm{Sb}_{\mathrm{x}}$ infrared detectors," Prog. Quantum Electron., vol. 13, no. 3, pp. 191-231, 1989, doi: 10.1016/0079-6727(89)900037. 
[4] M. Razeghi and B.-M. Nguyen, "Advances in mid-infrared detection and imaging: A key issues review," Rep. Prog. Phys., vol. 77, no. 8, p. 082401, Aug. 2014.

[5] A. Glozman et al., "High performance InAlSb MWIR detectors operating at $100 \mathrm{~K}$ and beyond," Proc. SPIE, vol. 6206, p. 62060M, May 2006, doi: $10.1117 / 12.667783$.

[6] D. Gibson and C. MacGregor, "A novel solid state non-dispersive infrared $\mathrm{CO}_{2}$ gas sensor compatible with wireless and portable deployment," Sensors, vol. 13, no. 6, pp. 7079-7103, May 2013, doi: $10.3390 / \mathrm{s} 130607079$.

[7] P. Capper and C. T. Elliott, Eds., Infrared Detectors and Emitters. Materials and Devices (Electronic Materials Series), vol. 8. New York, NY, USA: Springer, 2001, doi: 10.1007/978-1-4615-1607-1.

[8] Y. T. Gau et al., " $256 \times 256$ InSb focal plane arrays," Proc. SPIE, vol. 4078, p. 467, Jul. 2000, doi: 10.1117/12.392175

[9] H. S. Gupta et al., "Design of large dynamic range, low-power, highprecision ROIC for quantum dot infrared photo-detector," Electron. Lett., vol. 49, no. 16, pp. 1018-1020, Aug. 2013, doi: 10.1049/el.2013.1534.

[10] L.-W. Zhang, M. Shao, X.-L. Zhang, Q.-D. Meng, and J.-C. Wang, "Three-dimensional modeling and simulation of large-format hybrid indium antimonide detector arrays," Opt. Eng., vol. 52, no. 10, p. 103110 , Oct. 2013

[11] C. Xie et al., "Monolithic integration of an active InSb-based midinfrared photopixel with a GaAs MESFET," IEEE Trans. Electron Devices, vol. 62, no. 12, pp. 4069-4075, Dec. 2015.

[12] S. D. Wu et al., "Effect of the low-temperature buffer thickness on quality of $\mathrm{InSb}$ grown on GaAs substrate by molecular beam epitaxy," J. Crystal Growth, vol. 277, nos. 1-4, pp. 21-25, Apr. 2005, doi: $10.1016 /$ j.jcrysgro.2004.12.141

[13] K. Ueno et al., "InSb mid-infrared photon detector for room-temperature operation," Jpn. J. Appl. Phys., vol. 52, no. 9R, p. 092202, 2013

[14] M. Levinshtein, S. Rumyantsev, and M. Shur, Handbook Series on Semiconductor Parameters, vol. 1. Singapore: World Scientific, 1996.

[15] H. D. Trinh et al., "Electrical characteristics of $\mathrm{Al}_{2} \mathrm{O}_{3} / \mathrm{InSb}$ MOSCAPs and the effect of postdeposition annealing temperatures," IEEE Trans. Electron Devices, vol. 60, no. 5, pp. 1555-1560, May 2013, doi: 10.1109/TED.2013.2254119.

[16] C. Seok et al., "Raman spectroscopy of the damages induced by Ar-ion beam etching of $\mathrm{InSb}(100)$ surface," ECS Solid State Lett., vol. 3, no. 3, pp. P27-P29, Dec. 2014.

[17] J. Moore, H. Hendriks, and A. Morales, "Characterization and control of galvanic corrosion during GaAs wafer photoresist processing," in Proc. Int. Conf. Compound Semiconductor Manuf. Technol. (GaAs MANTECH), 2003. paper 8.8 .

[18] K.-M. Chang, J.-J. Luo, C.-D. Chiang, and K.-C. Liu, "Wet etching characterization of InSb for thermal imaging applications," Jpn. J. Appl. Phys., vol. 45, no. 3A, pp. 1477-1482, 2006.

[19] A. Tevke, C. Besikci, C. Van Hoof, and G. Borghs, "InSb infrared p-i-n photodetectors grown on GaAs coated Si substrates by molecular beam epitaxy," Solid-State Electron., vol. 42, no. 6, pp. 1039-1044, Jun. 1998, doi: 10.1016/S0038-1101(98)00124-5.

[20] Y. F. Lao et al., "Analysis of dark current mechanisms for splitoff band infrared detectors at high temperatures," IEEE Trans. Electron Devices, vol. 57, no. 6, pp. 1230-1236, Jun. 2010, doi: 10.1109/TED.2010.2046065.

[21] L. Meriggi et al., "Enhanced emission from mid-infrared AlInSb lightemitting diodes with p-type contact grid geometry," J. Appl. Phys. vol. 117, no. 6, p. 063101, Feb. 2015.

[22] A. R. Beattie, "Impact ionisation and quantum efficiency in InSb," Phys. Status Solidi B, vol. 111, no. 1, pp. 141-153, 1982, doi: $10.1002 / \mathrm{pssb} .2221110115$.

[23] G. K. Reeves, "Specific contact resistance using a circular transmission line model," Solid-State Electron., vol. 23, no. 5, pp. 487-490, May 1980, doi: 10.1016/0038-1101(80)90086-6.

[24] S. Donati, Photodetectors: Devices, Circuits and Applications. Upper Saddle River, NJ, USA: Prentice-Hall, Oct. 1999.

[25] J. T. Wimmers and D. S. Smith, "Characteristics of InSb photovoltaic detectors at 77K and below," Proc. SPIE, vol. 0364, p. 123, Aug. 1983 , doi: 10.1117/12.934189.

[26] I. Kimukin, N. Biyikli, and E. Ozbay, "InSb high-speed photodetectors grown on GaAs substrate," J. Appl. Phys., vol. 94, no. 8, p. 5414, 2003.

[27] I. Kimukin, N. Biyikli, T. Kartaloglu, O. Aytur, and E. Ozbay, "High-speed InSb photodetectors on GaAs for mid-IR applications," IEEE J. Sel. Topics Quantum Electron., vol. 10, no. 4, pp. 766-770, Jul./Aug. 2004.
[28] S. L. Solomon, J. D. Garnett, and H. Chen, "Investigation of chargetrapping effects in InSb focal plane arrays," Proc. SPIE, vol. 1946, p. 33 , Oct. 1993, doi: 10.1117/12.158702

[29] R. G. Benson, W. J. Forrest, J. L. Pipher, W. J. Glaccum, and S. L. Solomon, "Spatial distributions of hole traps and image latency in InSb focal plane arrays," Proc. SPIE, vol. 4131, p. 171, Nov. 2000 doi: $10.1117 / 12.406541$

[30] E. G. Camargo, S. Tokuo, H. Goto, and N. Kuze, "Nondispersive infrared gas sensor using InSb-based photovoltaic-type Infrared Sensor," Sensors Mater., vol. 26, no. 4, pp. 253-262, 2014.

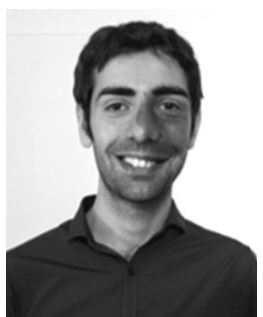

Vincenzo Pusino received the M.Sc. degree in electronics and electrical engineering from the University of Pavia, Pavia, Italy, in 2008, and the $\mathrm{Ph} . \mathrm{D}$. degree from the School of Engineering, University of Glasgow, Glasgow, U.K, in 2014.

$\mathrm{He}$ is currently a Research Assistant with the University of Glasgow. His current research interests include mid-IR imaging, III-V semiconductors, semiconductor mode-locked lasers, and integrated nonlinear optics.

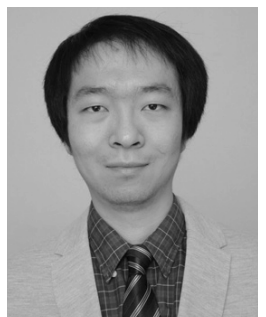

Chengzhi Xie received the B.Eng. degree from the College of Precision Instrument and Opto-Electronics Engineering, Tianjin University, Tianjin, China, in 2012. He is currently pursuing the $\mathrm{Ph} . \mathrm{D}$. degree with the University of Glasgow, Glasgow, U.K., with a focus on the integration of antimonide-based detectors and GaAs readout circuits for the implementation of new type of monolithic MWIR image sensor

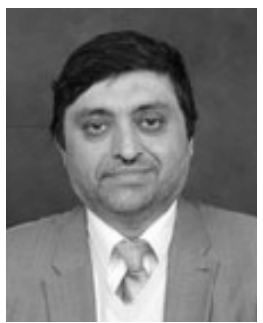

Ata Khalid (M'99) received the Ph.D. degree in electronic device engineering from King's College London, London, U.K., in 2000.

$\mathrm{He}$ was with King's College London several years to develop the MMIC design and fabrication processes and development of HBTs and HEMTs. In 2004, he joined the School of Engineering, University of Glasgow, Glasgow, U.K., to develop the solid-state $\mathrm{THz}$ radiation source technology based on planar Gunn diodes.

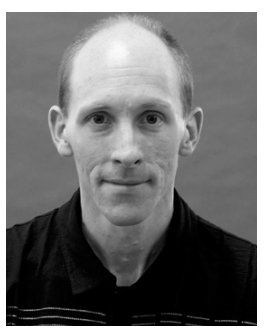

Matthew J. Steer received the B.Sc. degree in physics and the Ph.D. degree from the University of Sheffield, Sheffield, U.K.

$\mathrm{He}$ is currently a Research Associate with the University of Glasgow, Glasgow, U.K., where he is involved in the molecular beam epitaxy of III-V semiconductor materials for gas sensing applications.

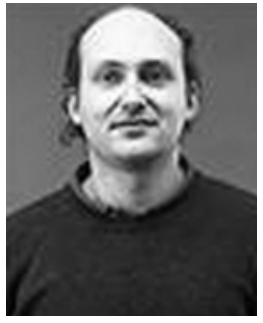

Marc Sorel received the M.Sc. degree in electronics engineering and the Ph.D. degree from the University of Pavia, Pavia, Italy, in 1995 and 1999. respectively.

He joined the Optoelectronics Group, University of Glasgow, Glasgow, U.K., in 1998, where he was a Professor in 2015. His current research interests include semiconductor technologies for monolithic and hybrid integration. 


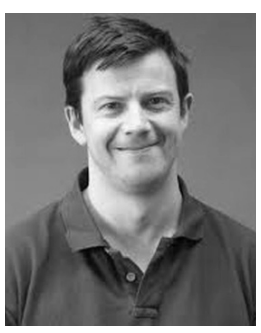

Iain G. Thayne received the B.Sc. and Ph.D. degrees from Glasgow University, Glasgow, U.K.

He was a Professor with the Ultrafast Systems Group, Glasgow University, in 2003. His current research interests include compound semiconductor MOSFETs for digital and $\mathrm{RF} /$ mixed signal applications and short gate length HEMTs and MMICs for millimeter-wave imaging and ultralow power distributed sensor network applications.

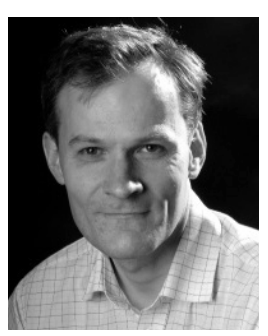

David R. S. Cumming (F'13) received the B.Eng. degree from the Glasgow University, Glasgow, U.K., and the Ph.D. degree from the University of Cambridge, Cambridge, U.K.

$\mathrm{He}$ is a Professor of Electronic Systems with Glasgow University, where he is also the Research Dean and leads the Microsystem Technology Group. $\mathrm{He}$ is involved in sensor systems and technologies for biomedical and imaging. 